\title{
Optical Potentials in Kaonic Atoms
}

\author{
Carmen García-Recio*, Juan Nieves*, Eulogio Oset ${ }^{\dagger}$ and Angels Ramos** \\ * Departamento Física Moderna, University of Granada, E-18071 Granada, Spain \\ ${ }^{\dagger}$ Departamento de Física Teórica and IFIC, Centro Mixto Universidad de Valencia-CSIC, Paterna, \\ Aptdo. Correos 22085, 46071 Valencia, Spain \\ ${ }^{* *}$ Departament d'Estructura i Constituents de la Matèria, Universitat de Barcelona, 08028
}

Barcelona, Spain

\begin{abstract}
A microscopic optical potential based on a chiral model is used as a starting point for studying kaonic atoms levels. We add to this potential a phenomenological part fitted to the experimentally known shifts and widths of kaonic levels. This fitted potential is used to predict deeply bound atomic levels, as well as nuclear levels. Comparison with the predictions of other optical models found in the literature is done. Also the effects on the kaonic atoms levels of certain known non-local contributions to the optical potential are also analyzed.
\end{abstract}

\section{THE SELFCONSISTENT $K^{-}$SELFENERGY OF RAMOS-OSET}

The problem of kaonic atoms has regained interest recently. First, due to the new perspective that the use of chiral Lagrangians has brought into the problem [1]. Second, because of the need to obtain accurately the kaon selfenergy in a nuclear medium, in view of the possibility to get kaon condensates in neutron-proton stars. Third, the interpretation of the enhancement of the $K^{-}$yields in heavy ion reactions relies on the value of the $K^{-}$selfenergy in the nuclear medium.

The dominance of the s-wave in the elementary $\bar{K} N$ interaction has been the justification for using traditionally s-wave $\bar{K}$ nucleus optical potentials [2,3], by means of which good agreement with data can be obtained. In the work of Ramos-Oset [1], the s-wave self-energy $\Pi_{\bar{K}}^{S}$ of the $K^{-}$meson in nuclear matter is calculated in a selfconsistent microscopic approach, using an in medium effective $\bar{K} N$ interaction, $t_{i j}^{e f f}$, obtained from the lowest-order meson-baryon chiral lagrangian $V_{i j}$ by solving the coupled-channel BetheSalpeter equation for the meson-baryon sector with strangeness $S=-1$. For our calculations of shifts and widths in kaonic atoms, we take as starting point this local theoretical potential, $V_{\mathrm{opt}}^{(1)}$ (dashed line in fig. 1). We also use an improved version of this potential, $V_{\mathrm{opt}}^{\left(1_{\Sigma^{*}}\right)}$ (shown in fig. 1 with solid line), which includes the $\Sigma^{*} h$-excitation in the selfconsistent calculation of the s-wave $K^{-}$optical potential.

\section{CALCULATION OF SHIFTS AND WIDTHS OF KAONIC ATOMS}

Purely s-wave potential. By using the s-wave potentials $(1)$ and $\left(1_{\Sigma^{*}}\right)$ we calculate the shifts and widths corresponding to 63 experimental data (see table 1) obtaining 

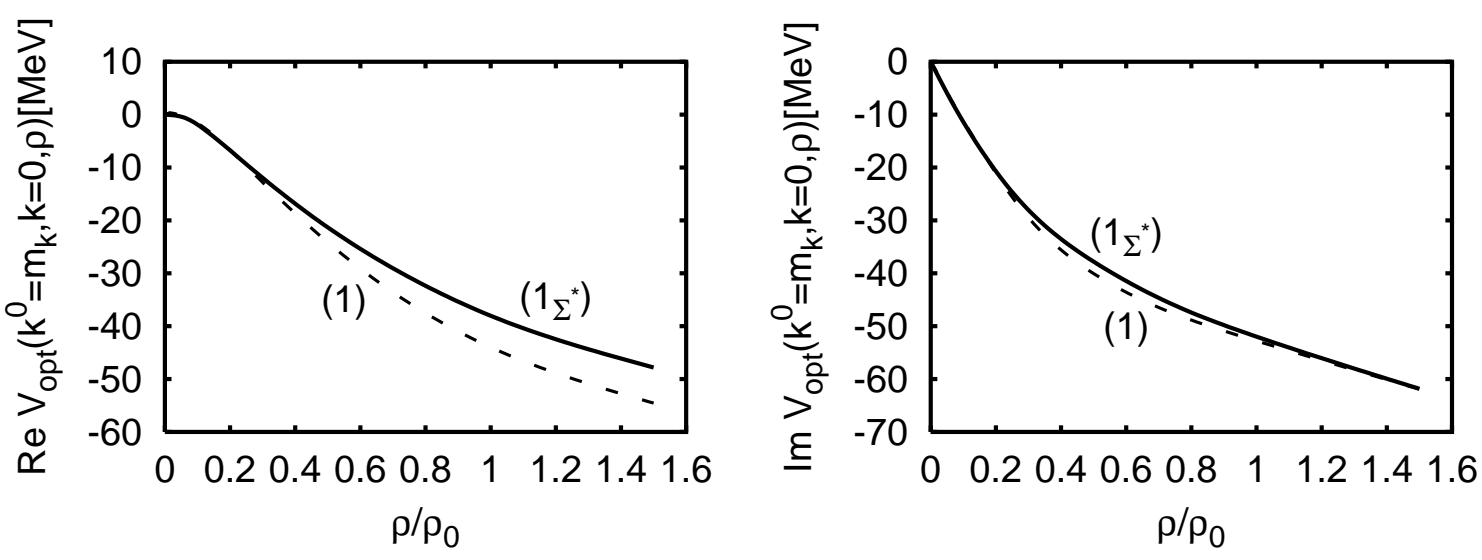

FIGURE 1. $K^{-}$optical potentials versus nuclear density.

values of $\chi^{2}$ per data of 3.76 and 2.89 , respectively. The agreement with the data is quite satisfactory if one takes into account that the potentials are purely theoretical without any free parameter.

p-wave. The lowest order p-wave optical potential [4] includes the lowest order p-wave chiral lagrangian plus the contribution of the $\Lambda h, \Sigma h$ and $\Sigma^{*} h$ excitations. Using the s-wave potential $V_{\text {opt }}^{(1)}$ plus this p-wave part to solve the Klein-Gordon equation, we find the results of row (2) in table 1 , with $\chi^{2} / N=4.00$. We observe that the change in the shifts and widths due to the p-wave are much smaller than the experimental uncertainties.

s-wave induced non-local effects. The s-wave optical potential of a $K^{-}$in a nuclear medium of density $\rho$ depends on the $K^{-}$energy $\omega$ and momentum $\vec{k}$. For kaonic atoms, the potentials (1) and $\left(1_{\Sigma^{*}}\right)$ were evaluated at threshold $\left(\omega=m_{K}, \vec{k}=0\right)$. Now, we consider the corrections to the optical potential due to the explicit $\omega$ and $\vec{k}$ dependence. Expanding the $K^{-}$selfenergy at first order around threshold:

$$
\Pi(\omega, \vec{k}, \rho)=2 \omega V_{\mathrm{opt}}=\Pi\left(m_{K}, 0, \rho\right)+b(\rho) \vec{k}^{2}+c(\rho)\left(\omega-m_{K}\right)
$$

The momentum $\vec{k}$ is not defined for a $K^{-}$bound in an atom and instead it becomes an operator. Having evaluated the selfenergy in nuclear matter, it is not well defined which kind of $\vec{\nabla} \vec{\nabla}$ operator will correspond to the factor $\vec{k}^{2}$. So different realizations for the operator $\vec{k}^{2}$ have been considered, see rows (3a) and (3b) in table 1 . The effect of considering the $c\left(\omega-m_{K}\right)$ term is shown in row (4) of the table. We observe that the effect of any of these non local terms on shifts and widths are smaller that the error bars of the experimental data. For more details on non-local effects see ref. [4]. 
TABLE 1. Widths and shifts of representative kaonic atom levels in eV obtained from different potentials. Also $\chi^{2}$ per number of data, $N=63$, is shown. Row (1) correspond to the local potential of ref. [1]. Rows (2) to (4) correspond to different non-local additions to this dominant piece (1):

(2) Only p-wave non-local effects due to the coupling of $K^{-} N$ to $\Lambda, \Sigma$ and $\Sigma^{*}$, are added.

(3) Only lowest order in momentum $b \vec{q}^{2}$ non-local terms of the s-wave potential, see eq. (1), are included in two different ways: (3a) $-\vec{\nabla} b \vec{\nabla},(3 \mathrm{~b})-\vec{\nabla} b \vec{\nabla}-0.5(\Delta b)$.

(4) Only energy dependent $c(\omega-\mu)$ "non-local" effects of eq. (1) are added.

The results of row $\left(1_{\Sigma^{*}}\right)$ are obtained from a theoretical $s$-wave optical potential, like in row (1), but including $\Sigma^{*} h$ excitations. Potentials $(1 \mathrm{~m}),\left(1_{\Sigma^{*}} b_{0}\right)$ and $\left(1_{\Sigma^{*}} B_{0}\right)$ are best-fit local potentials.

\begin{tabular}{|c|c|c|c|c|c|c|c|c|c|c|c|}
\hline & \multirow[t]{2}{*}{$\chi^{2} / N$} & \multicolumn{2}{|c|}{${ }_{5}^{10} \mathrm{~B}$} & \multicolumn{2}{|c|}{${ }_{13}^{27} \mathrm{Al}$} & \multicolumn{2}{|c|}{${ }_{29}^{63} \mathrm{Cu}$} & \multicolumn{2}{|c|}{${ }_{48}^{112} \mathrm{Cd}$} & \multicolumn{2}{|c|}{${ }_{92}^{238} \mathrm{U}$} \\
\hline & & $-\varepsilon_{2 p}$ & $\Gamma_{2 p}$ & $-\varepsilon_{3 d}$ & $\Gamma_{3 d}$ & $-\varepsilon_{4 f}$ & $\Gamma_{4 f}$ & $-\varepsilon_{5 g}$ & $\Gamma_{5 g}$ & $-\varepsilon_{7 i}$ & $\Gamma_{7 i}$ \\
\hline (1) & 3.76 & 217 & 551 & 109 & 368 & 384 & 1121 & 528 & 1437 & 330 & 1090 \\
\hline (2) & 4.00 & 213 & 542 & 110 & 362 & 392 & 1110 & 543 & 1420 & 350 & 1076 \\
\hline (3a) & 3.20 & 211 & 565 & 102 & 397 & 361 & 1229 & 494 & 1588 & 302 & 1291 \\
\hline (3b) & 4.00 & 234 & 564 & 118 & 383 & 415 & 1172 & 568 & 1515 & 357 & 1196 \\
\hline (4) & 3.69 & 217 & 552 & 110 & 371 & 388 & 1141 & 534 & 1465 & 337 & 1166 \\
\hline$\left(1_{\Sigma^{*}}\right)$ & 2.89 & 208 & 575 & 105 & 398 & 373 & 1219 & 512 & 1550 & 320 & 1201 \\
\hline$(1 \mathrm{~m})$ & 1.52 & 159 & 742 & 69 & 438 & 335 & 1290 & 490 & 1610 & 270 & 1100 \\
\hline$\left(1_{\Sigma^{*}} b_{0}\right)$ & 1.30 & 156 & 722 & 68 & 449 & 309 & 1368 & 453 & 1732 & 255 & 1241 \\
\hline$\left(1_{\Sigma^{*}} B_{0}\right)$ & 1.41 & 148 & 673 & 70 & 438 & 308 & 1386 & 448 & 1781 & 279 & 1297 \\
\hline Exp & - & $\begin{array}{r}208 \\
\pm 35\end{array}$ & $\begin{array}{r}810 \\
\pm 100\end{array}$ & $\begin{array}{r}80 \\
\pm 13\end{array}$ & $\begin{array}{r}443 \\
\pm 22\end{array}$ & $\begin{array}{r}370 \\
\pm 47\end{array}$ & $\begin{array}{r}1370 \\
\pm 170\end{array}$ & $\begin{array}{r}400 \\
\pm 100\end{array}$ & $\begin{array}{r}2010 \\
\pm 440\end{array}$ & $\begin{array}{r}260 \\
\pm 400\end{array}$ & $\begin{array}{r}1500 \\
\pm 750\end{array}$ \\
\hline
\end{tabular}

\section{FITS TO ATOMIC DATA}

By adding a fitted correction $\delta V^{\text {fit }}$ to the previous theoretical optical potentials, we achieve theoretically founded phenomenological potentials that describe the experimental data quite satisfactorily. We consider two different forms for the fitted part: $2 \mu \delta V_{b_{0}}^{\text {fit }}(r)=-4 \pi\left(1+\mu / M_{N}\right) \rho(r) \delta b_{0}$, with one complex parameter $\delta b_{0}$, and $2 \mu \delta V_{B_{0}}^{\mathrm{fit}}(r)=-4 \pi\left(1+\mu / M_{N}\right) \rho(r)\left(\rho(r) / \rho_{0}\right)^{1 / 3}\left(i \delta \operatorname{Im} B_{0}\right)$, with one real parameter $\delta \operatorname{Im} B_{0}$. The quantity $\mu$ is the $K^{-}$-nucleus reduced mass. The phenomenological potential $(1 \mathrm{~m})$ is obtained from (1) plus $\delta V_{b_{0}}^{\text {fit }}$ with $\delta b_{0}=(0.078-i 0.25) \mathrm{fm}$. The potential

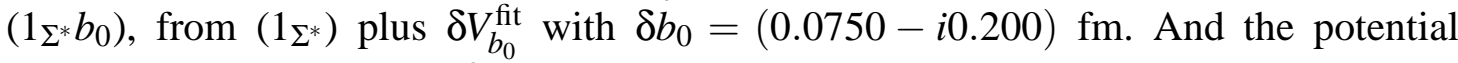
$\left(1_{\Sigma^{*}} B_{0}\right)$, from $\left(1_{\Sigma^{*}}\right)$ plus $\delta V_{B_{0}}^{\text {fit }}$ with $\delta \operatorname{Im} B_{0}=-0.260 \mathrm{fm}$. They provide good fits with $\chi^{2}$ per data of about 1.4 , see rows $(1 \mathrm{~m}),\left(1_{\Sigma^{*}} b_{0}\right)$ and $\left(1_{\Sigma^{*}} B_{0}\right)$ of table 1 .

Predictions: deeply-bound atomic levels. The fitted potential $(1 \mathrm{~m})$, described above, is used to predict binding energies and widths of deeply bound atomic states, not yet observed. They are shown, for ${ }^{208} \mathrm{~Pb}$, in fig. 2. See ref. [3] for other nuclei. One can see that the levels, including the widths, do not overlap for a given angular momentum. 


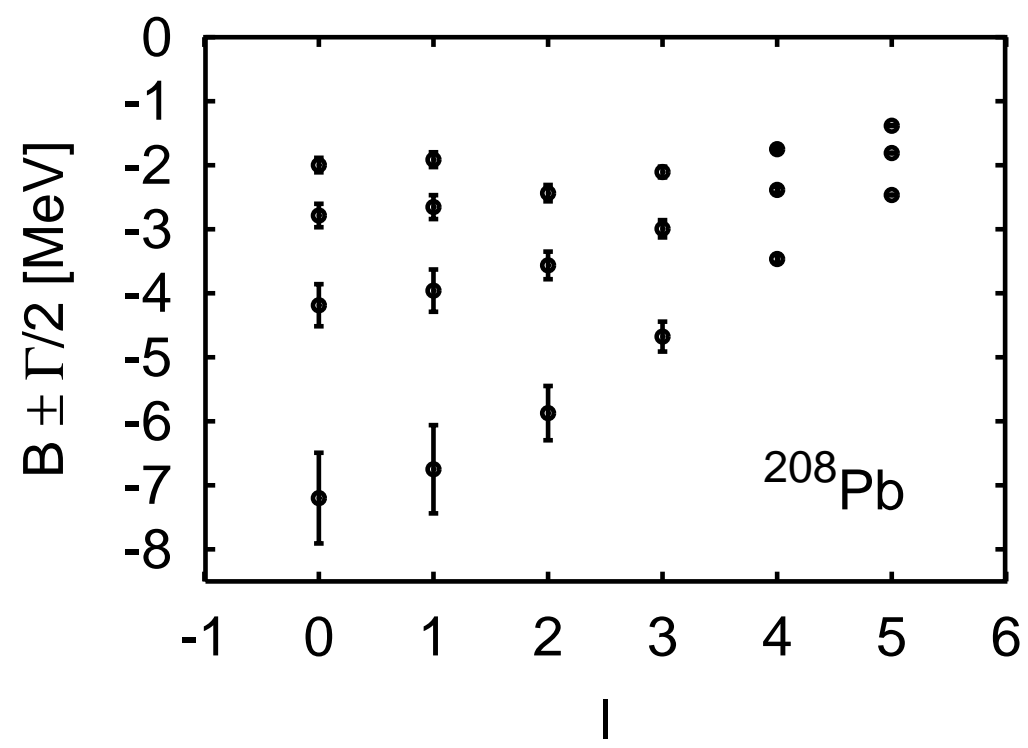

FIGURE 2. Binding energies $B$ of deeply bound atomic levels in ${ }^{208} \mathrm{~Pb}$ versus angular momentum $l$. The error bar stands for the full width $\Gamma$ of each level. They have been computed using the $V_{\mathrm{opt}}^{(1 \mathrm{~m})}$ potential.

\section{CONCLUSIONS}

- The selfconsistent microscopic approach based on the chiral lagrangian $\left(1_{\Sigma^{*}}\right)$ is quite good: $\chi^{2} / N=2.9$, for 63 atomic data, with no free parameter in the model.

- Non-local effects, associated to proper p-wave contributions or to momentum and energy dependence of the s-wave self-energy, are negligible at this stage, because their effect on shifts and widths are smaller than the current uncertainties of data.

- An improved fitted potential, $\left(1_{\Sigma^{*}} b_{0}\right)$, provides a very good fit with $\chi^{2} / 63=1.3$.

- Deeply-bound kaonic atom levels are narrow and separable, so subjected to experimental observation via nuclear reactions. The widths of these levels are sensitive to different potentials by about a $20 \%$.

\section{ACKNOWLEDGMENTS}

This work was partially supported by DGICYT contract PB98-1367 and Junta de Andalucía under grant FQM 0225

\section{REFERENCES}

1. A. Ramos and E. Oset, Nucl. Phys. A671 (2000) 481 and references therein.

2. E. Friedman and A. Gal, Phys. Lett. B459 (1999) 43.

3. A. Baca, C. García-Recio, J. Nieves Nucl. Phys. A673 (2000) 335.

4. C. García-Recio, J. Nieves, E. Oset and A. Ramos, nucl-th/0012075. 